\title{
Expansion of a $Y$-Shaped Antenna Array and Optimization of the Future Antenna Array in Malaysia for Astronomical Applications
}

\author{
Shahideh Kiehbadroudinezhad' ${ }^{1}$ Jean-François Bousquet ${ }^{1}$, Michael Cada1 ${ }^{1}$ C. Ian Short ${ }^{2}$, \\ Adib Shahabi1 ${ }^{*}$, Samiramis Kiehbadroudinezhad ${ }^{3,4}$ \\ ${ }^{1}$ Department of Electrical and Computer Engineering, Faculty of Engineering, Dalhousie University, Halifax, Canada \\ ${ }^{2}$ Department of Astronomy and Physics, Saint Mary's University, Halifax, Canada \\ ${ }^{3}$ Department of Physics, Eberhard-Karls-Universität Tübingen, Tübingen, Germany \\ ${ }^{4}$ Department of Astronomy, Institute of Astronomy and Astrophysics, Tübingen, Germany \\ Email: *adib.shahabi@dal.ca
}

How to cite this paper: Kiehbadroudinezhad, S., Bousquet, J.-F., Cada, M., Short, C.I., Shahabi, A. and Kiehbadroudinezhad, S. (2019) Expansion of a $Y$-Shaped Antenna Array and Optimization of the Future Antenna Array in Malaysia for Astronomical Applications. Journal of Modern Physics, 10, 888-908.

https://doi.org/10.4236/jmp.2019.108059

Received: May 26, 2019

Accepted: July 2, 2019

Published: July 5, 2019

Copyright $\odot 2019$ by author(s) and Scientific Research Publishing Inc. This work is licensed under the Creative Commons Attribution International License (CC BY 4.0).

http://creativecommons.org/licenses/by/4.0/

\begin{abstract}
To achieve high quality images from the sky by extending an existing interferometric array, in this work, the Geometrical Method (GM), Genetic Algorithm ( $G A$ ), and Division Algorithm $(D A)$ are compared. These methods are each applied independently to an interferometer array starting from the same initial conditions. Using the GM method, the spiral configuration is suggested as an optimum arrangement that provides the desired $u-V$ coverage with low side lobe levels ( $S L L s)$. Using the $G A$ method, as the number of generations is increased, the unsampled cells are reduced, enhancing the imaging quality. As such, the algorithm improves the overlapped samples as it works with a greater number of generations. Moreover, the $G A$ is able to suppress the $S L L$. Finally, the $D A$ is applied to such an array. Results show that the $D A$ is able to process the sampled data with less overlapping of the data in the snapshot observations, in comparison to the other discussed configurations in this paper; effectively the $D A$ reduces the overlapped samples, such that it is more efficient than the $G A$. The configuration of antennas that arrives by applying the $D A$ method can achieve a certain image quality with less overlapping, as compared to the configuration arriving by applying the $G A$ method. The calculated $S L L s$ for the $D A$ configuration are used to demonstrate that the efficiency of the $D A$ is potentially better than that of the $G A$. Moreover, the $G A$ and $D A$ algorithms discussed in this study are applied to an array of 10 antennas with coordinates that represent the antennas deployed in Malaysia. Results show that the $D A$ can reduce the overlapping of the samples more efficiently than the $G A$ for a 6-hour tracking observation and in terms of unsampled cells the $D A$ has the same efficiency of the $G A$.
\end{abstract}




\section{Keywords}

Interferometers, Telescopes, Genetic Algorithm, Array of Antennas

\section{Introduction}

In order to measure with fine angular detail in the radio frequency range from the sky, two-element radio interferometers, or alternatively synthesis arrays can be used. The angular resolution of a single telescope does not provide sufficient information for astronomy applications; therefore, a synthesis array or radio interferometer is used to fulfill the aim of the end user. On the other hand, the light waves from very distant stars or galaxies take a long time to travel through space to our telescopes; therefore, it limits the astronomers to visually observe light waves that occurred a very long time ago.

The time lag to observe events has led astronomers to build more powerful telescopes to study the first stars and galaxies formed. One of the most powerful arrays is the Giant Meterwave Radio Telescope (GMRT). To get higher resolution than what the current GMRT array can provide, additional antennas were added to the array. To improve existing correlator antenna arrays like the Giant Metrewave Radio Telescope (GMRT), an expansion of the array is required to obtain higher resolution. A project called the Square Kilometre Array $(S K A)$, which involves more than ten countries worldwide, will be the most powerful radio telescopes array. It allows observation of the sky and it can produce images from radio waves with very high resolution. However, the location of the telescope limits the image quality and has a direct effect on the side lobe levels (SLLs) [1].

In the design of an array for astronomy applications, the choice of each antenna's localization in the array is key. An ideal arrangement must ensure optimal configuration to capture a clear image of a radio source by either decreasing the side lobe level $(S L L)$ in the $1-m$ domain or increasing the sampled data in the spatial frequency domain, which is referred to as $u$ - $v$ plane coverage [2].

In this work, we focus on the comparison of different methods: the Geometrical Method ( $G M)$, the Genetic Algorithm $(G A)$, and the Division Algorithm (DA) [1] [2] [3] and [4]. These techniques and methods for assisted interferometry and how they can be implemented in a correlator antenna array, particularly applied to the $S K A$ are described. The ability of the proposed receiver to suppress the severe $S L L$ effect, to increase the $u-V$ plane coverage, and to smoothen the linear ridges in the $u-V$ plane coverage at a low number of snapshots or low duration of observation will be demonstrated through simulations, using Matlab.

The first method ( $G M)$ uses the optimization of the array configuration problem with various changes of coordinates in a specific area with the GMRT's arms as an illustrative example. The results show that spiral configurations give very 
good improvement in both $u-v$ plane coverage and reduction of side lobe levels.

For the second technique, the proposed $G A$ presented in [2] is used to optimize a correlator array of antennas. The algorithm is able to distribute the $u-V$ plane more efficiently than $G M R T$ with $49.77 \%$ overlapped samples. The configuration arrived at with this algorithm is able to sample the $u$ - $V$ plan more efficiently than the current GMRT configuration.

The third method is the new proposed algorithm named Division Algorithm $(D A)$ that has been recently presented in [3] to solve optimization problems.

The above methods are used to yield the optimum configuration for an extended interferometric array, and effectively to investigate the feasibility of extending the interferometric array using 10 antennas that would be deployed in Malaysia.

This paper is organized as such; Section 2 introduces the material and methods for expanding a $Y$-shape array and to represent the antenna array in Malaysia. Section 3 presents the simulation results. Finally, Section 4 and 5 cover the discussion and conclusions, respectively.

\section{Material and Methods}

\subsection{Expanded $Y$-Shape}

To extend the GMRT, it is proposed to add 15 antennas for cost-effectiveness and to realize the scientific goals. In order to add the antennas to the current array, the position of each antenna using rectangular coordinates $(x, y, z)$ is calculated using:

$$
\begin{gathered}
X=R \times \sin \theta \times \cos \varphi \\
Y=R \times \sin \theta \times \sin \varphi \\
Z=R \times \cos \theta
\end{gathered}
$$

where $R, \theta$, and $\varphi$ are the latitude, longitude, and radial distance $R$ (equal to about $6378.1 \mathrm{~km}$, the value of earth's radius), respectively. The extended configuration is placed at $110 \mathrm{~km}$ along the arms of the GMRT. The declination of the radio frequency source of interest for all configurations is the same (and is equal to $45^{\circ}$ ). Spiral configurations follow the theory of a logarithmic spiral [1].

Results in [1] show that the spiral antenna array configuration provides low $S L L$ and good $u-V$ coverages and therefore achieves high image resolution. Therefore, the following equations are used to calculate the gridded cells in $u-V$ domain:

$$
\begin{gathered}
N_{\text {grid }}=\sqrt{\left(\frac{A_{t}}{A}\right) \times(\text { nosmp })} \\
\Delta u=\frac{u_{\text {max }}-u_{\text {min }}}{N_{\text {grid }}} \\
\Delta v=\frac{v_{\text {max }}-v_{\text {min }}}{N_{\text {grid }}}
\end{gathered}
$$


where nosmp, $A_{t}, A$, and $N_{\text {grid }}$ are the number of samples in the snapshot or hour tracking observation, the total desired area, the covered area by the current configuration, and the number of gridded cells, respectively.

And $\Delta u, \Delta v, u_{\max }, u_{\min }, v_{\max }, v_{\min }$ and $N_{\text {grid }}$ are the dimension of the cell in the $u$ direction, the dimension of the cell in the $v$ direction, the maximum value of $u$ in the spatial frequency domain, the minimum value of $u$ in the spatial frequency domain, the maximum value of $v$ in the spatial frequency domain, the minimum value of $v$ in the spatial frequency domain and the number of gridded cells as defined in Equation (2), respectively [1].

Also, results in [2] show the good performance of the $G A$ configuration in terms of $S L L$ and sampling. The algorithm provides an optimum solution for both a snapshot observation and a 6-hour tracking observation with minimum values of overlapping. This happens due to the discrete grid locations of the plane as defined in:

$$
N_{\text {grid }}=\text { nearest } \sqrt{\left(n \times(n-1) \times A_{\text {total }} / A_{\text {first }}\right)}
$$

where $n$ is the number of antennas, $A_{\text {total }}$ is the total area, $A_{\text {first }}$ is the first calculated area of tracking observation from the first random population, and nearest rounds $N_{\text {grid }}$ to the nearest integer. This selection helps distribute the samples in the $u-v$ plane with less overlapping in both snapshot and hour tracking simultaneously [1].

The new algorithm $(D A)$, explained in [3], shows good results in optimizing an interferometric array. Therefore, these three methods are used in this section to investigate an optimum configuration, in order to extend an interferometric array.

To evaluate the position of each antenna using $G A$ and $D A$ methods, the fitness function elaborated in [2] is used:

$$
\text { fitness }(k)=-\frac{o l_{\text {sam }}(k)}{\max (o l)}-\frac{D(k)}{R}-\frac{\left(A_{\mathrm{tofsam}}-A_{\mathrm{sam}}(k)\right)}{A_{\mathrm{tofsam}}}
$$

where: fitness $(k)$ is the fitness value of $k^{\text {th }}$ baseline in $n^{\text {th }}$ generation. $o l_{\text {sam }}(k)$ indicates the number of overlapped samples generated by $k^{\text {th }}$ baseline in $n^{\text {th }}$ generation. $\operatorname{Max}(o l)$ provides the maximum value of overlapping that is in $n^{\text {th }}$ generation. $D(k)$ is the sample distance from the grid center. $R$ is the gridded cell radius. $A_{\text {sam }}(k)$ is the calculated area generated by $k^{\text {th }}$ baseline's samples in $n^{\text {th }}$ generation. $A_{\text {tofsam }}$ is the total area generated by $n^{\text {th }}$ generation [2].

Finally, the mean $S L L$ for all the aforementioned methods are calculated using the following equation [1]:

$$
\text { mean } S L L=- \text { mean }(\text { first } S L L+\text { second } S L L+\text { third } S L L)
$$

\subsection{Antenna Array in Malaysia}

Malaysia has started to fund major research astronomy projects recently with two telescopes. In this work, we apply the proposed theory of localization using 
an array of antennas for astronomy applications to suppress the SLLS and/or increase the number of samples in the $u-v$ plane coverage for the future array configuration in Malaysia.

We expect that the proposed methods can optimize the number of data samples and minimize the side lobe levels in the angular domain to enhance the image quality as much as possible. The methods discussed in this study are applied to 10 antennas which will be placed at the coordinates of the antennas in Malaysia.

The materials and methods used in this section are taken from [2] [3]. Here, the $G A$ and $D A$ algorithms are used to investigate the optimum solutions for ten antennas in Malaysia.

\section{Results}

\subsection{Expanded $Y$-Shape}

To expand the existing array, 15 antennas are added for the following configurations: 1) extended $Y$-shaped; 2) spiral; 3) $25^{\text {th }}$ generation using $G A$; 4) $150^{\text {th }}$ generation using $G A$; and 5) $D A$. Figure 1 shows the different configurations. The $u-V$ plane coverage achieved from these different configurations are shown in Figure 2 and Figure 3 for the snapshot and 6-hour tracking observations, respectively. The results are summarized in Figures 1-3, and Table $1 \&$ Table 2.

In the first step, extension of the $Y$-shaped array is investigated. In this configuration, 15 antennas are located along the three arms of the GMRT. Five antennas are added in each arm and broadened around to $110 \mathrm{~km}$. This array gets a $Y$-shape to investigate the effect of extending the arms in the original $Y$-shape. This new arrangement, its snapshot observation, and 6-hour image synthesis are shown in Figure 1(a), Figure 2(a), and Figure 3(a), respectively.

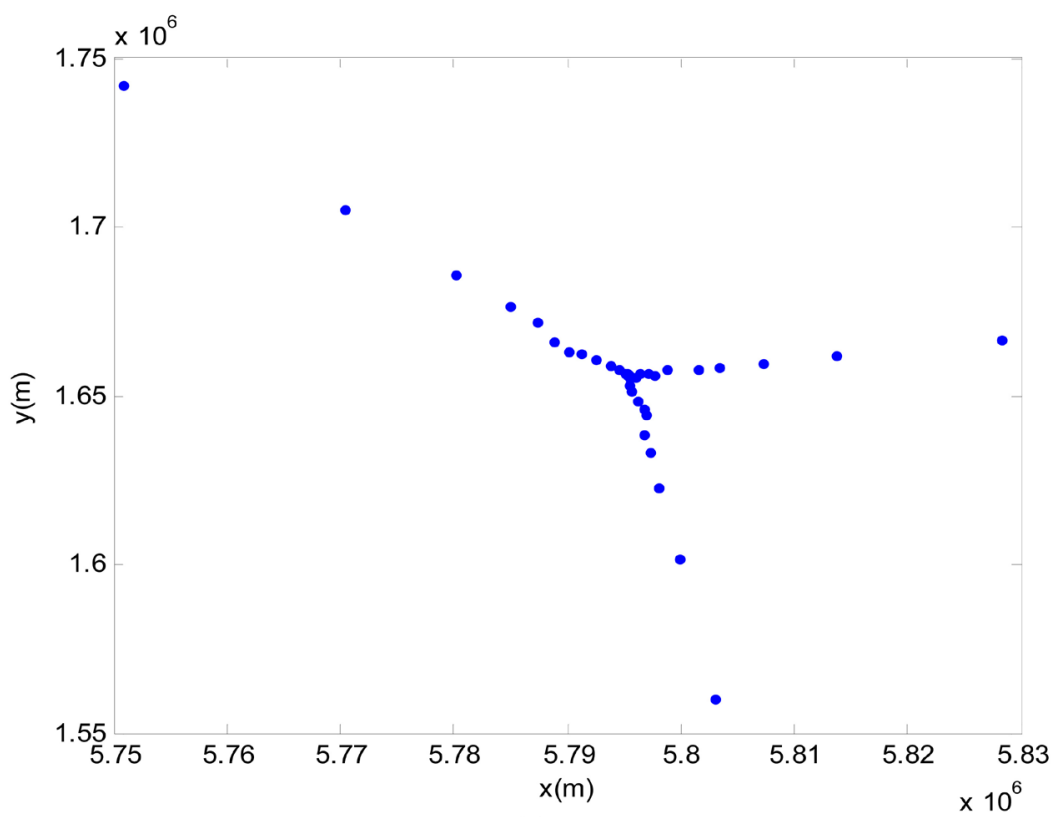

(a) 


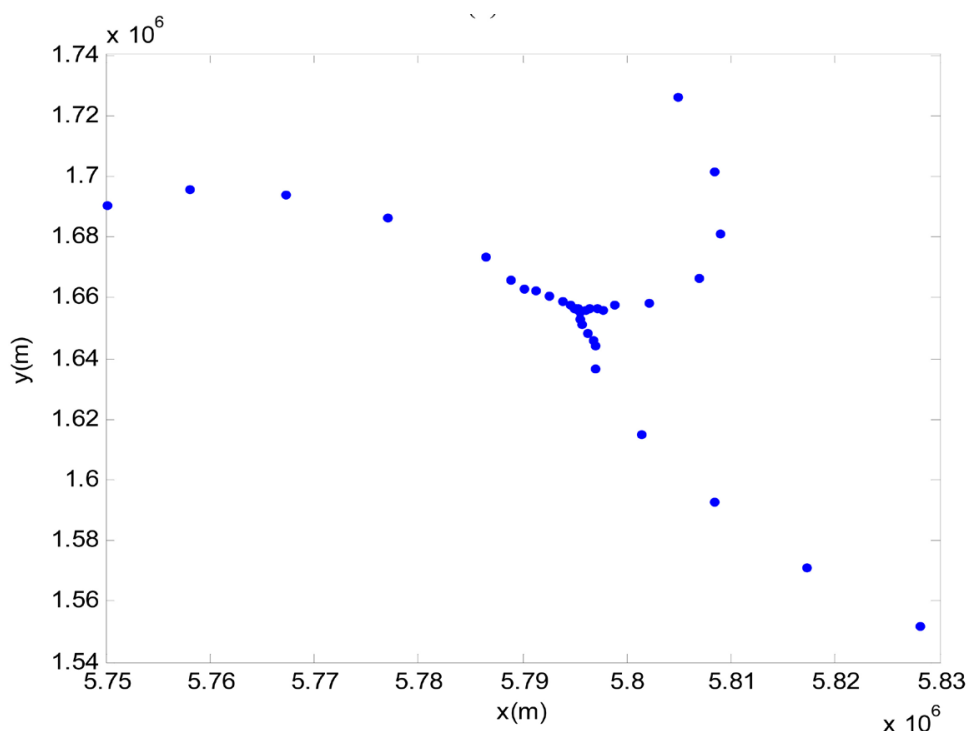

(b)

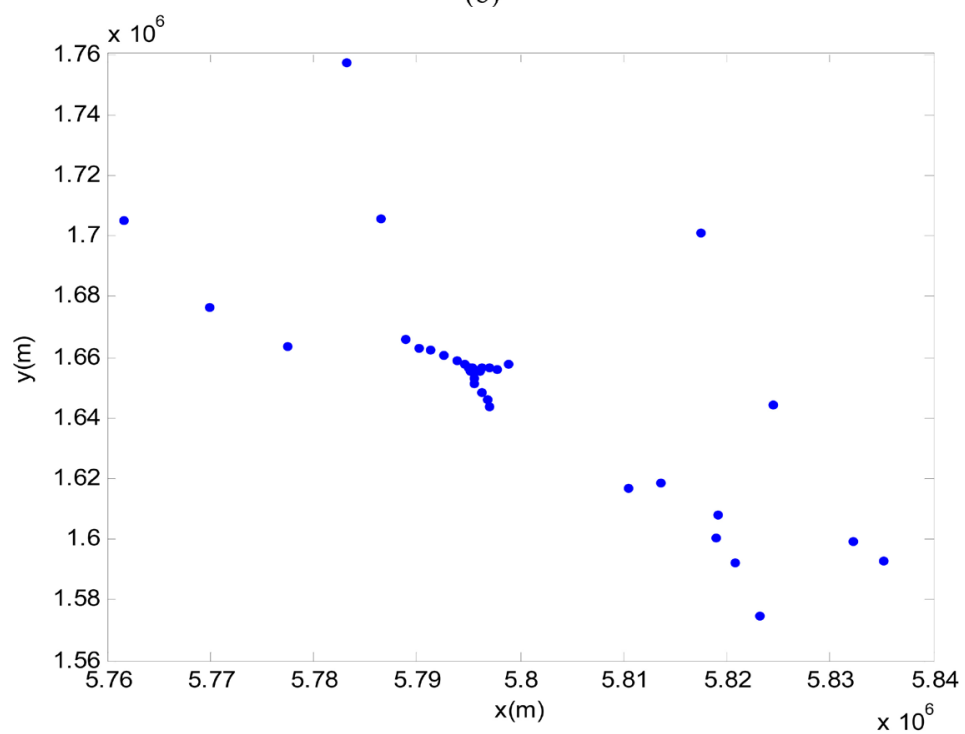

(c)

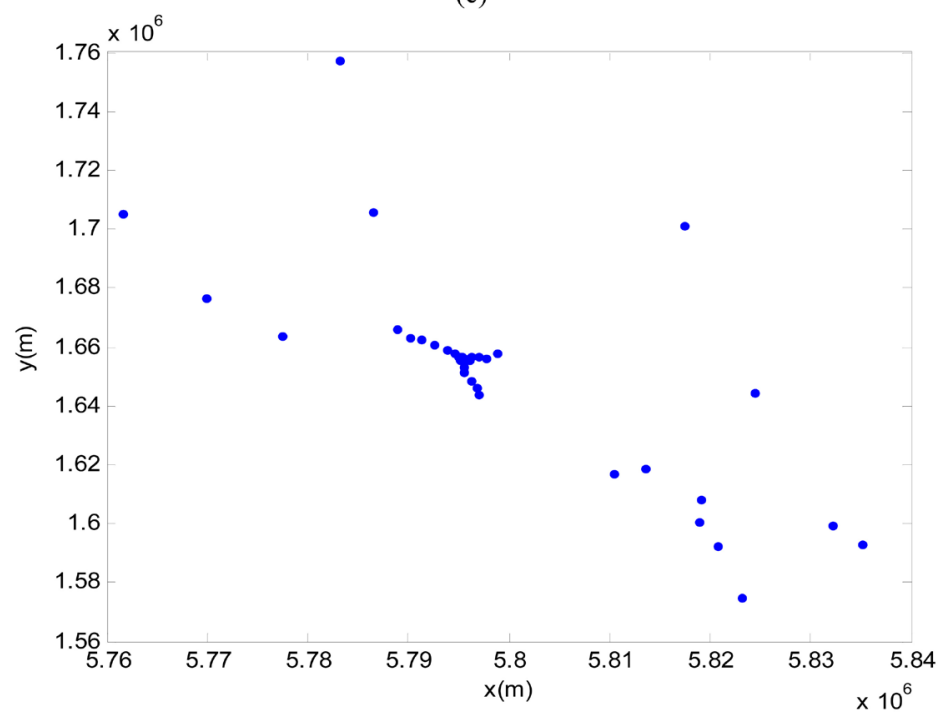

(d) 


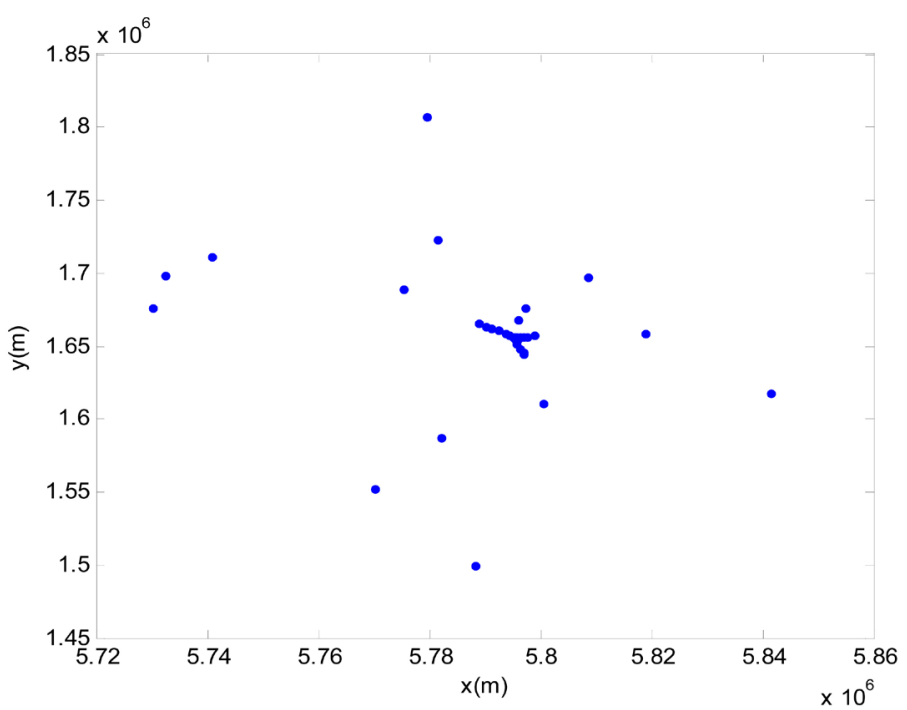

(e)

Figure. 1. Configuration for (a) extended $G M R T$, (b) spiral, (c) twenty-five generations, (d) one hundred fifty generations using Genetic Algorithm and (e) Division Algorithm.

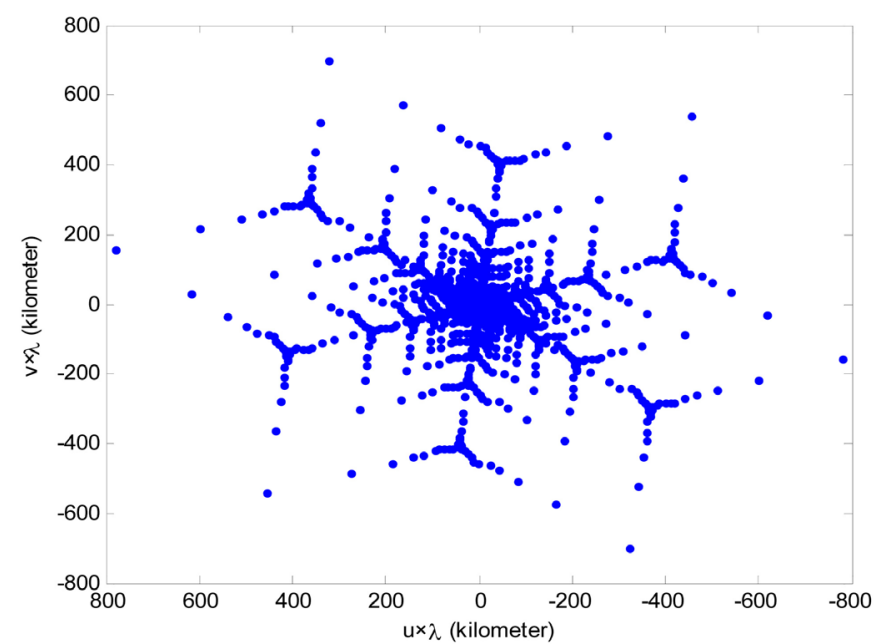

(a)

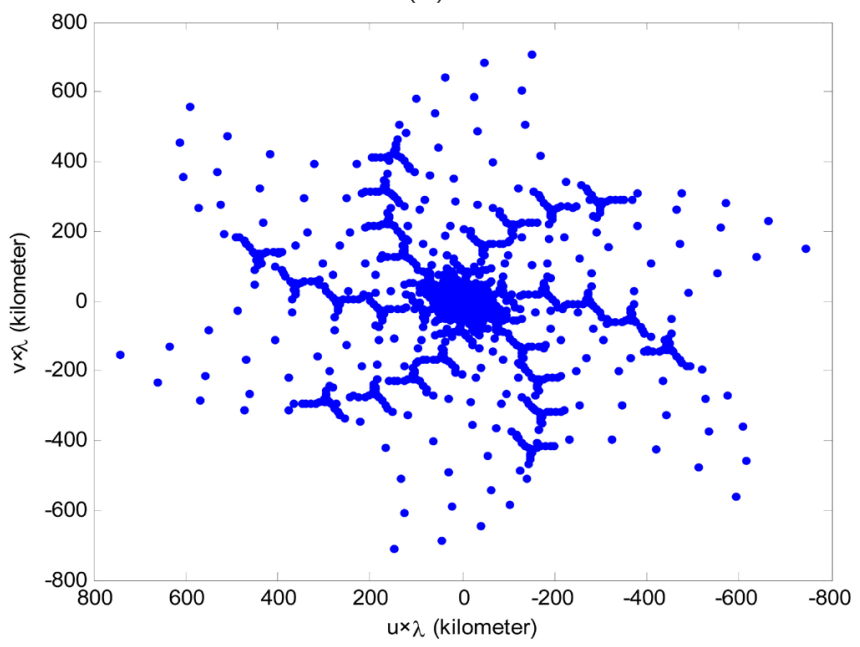

(b) 


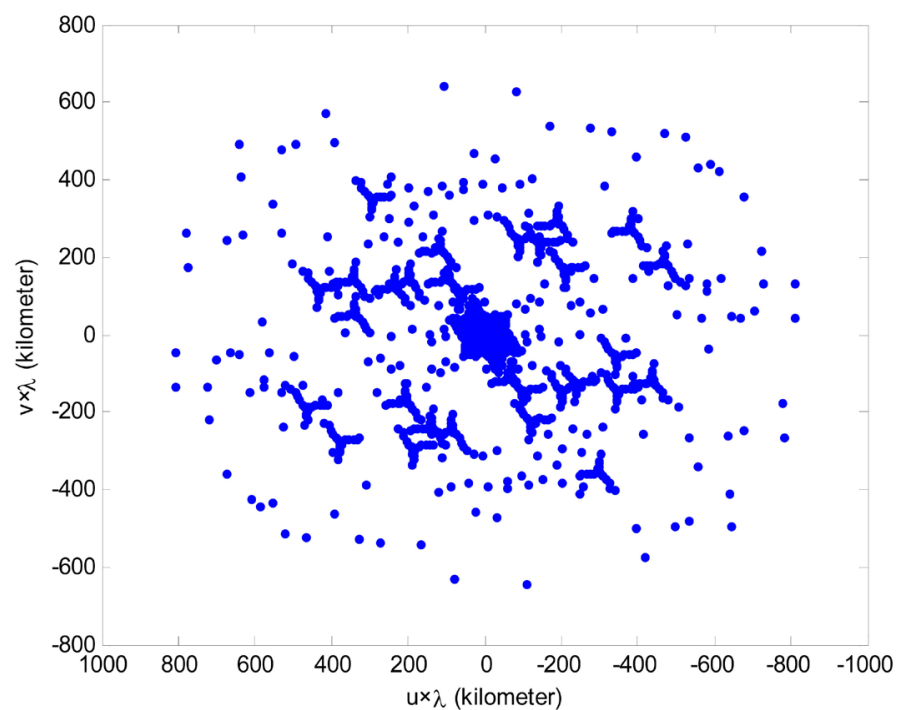

(c)

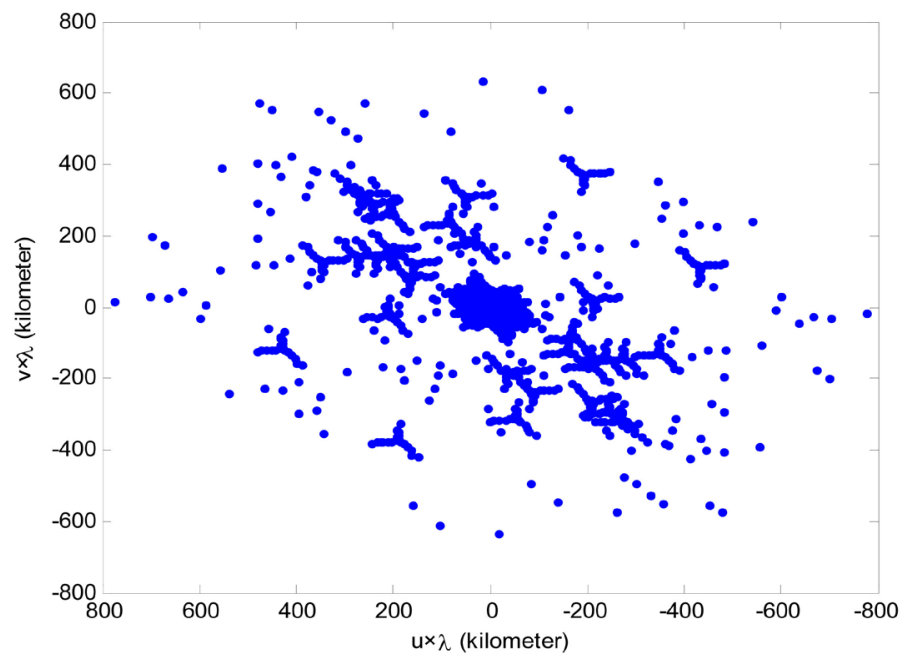

(d)

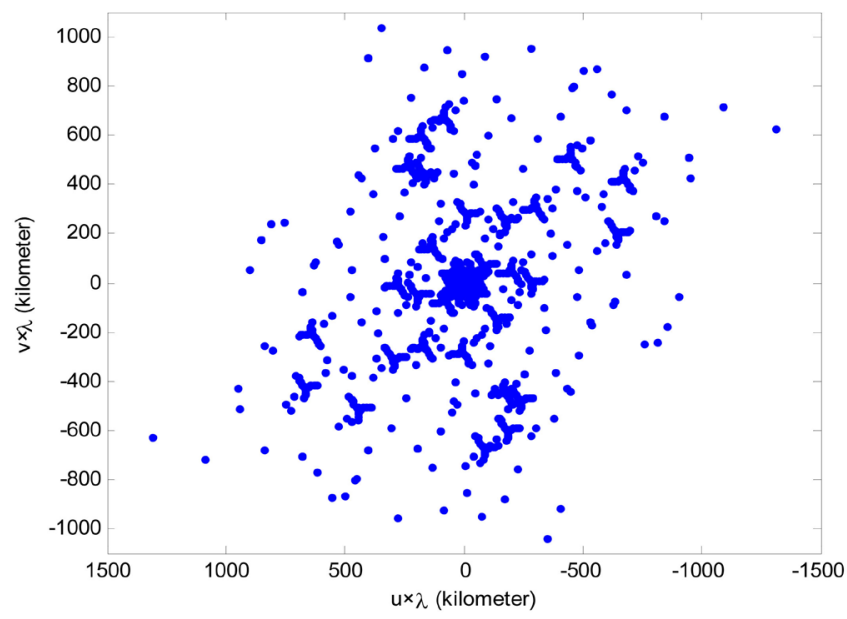

(e)

Figure 2. Snapshot $u$-V plane coverage for (a) extended $G M R T$, (b) configuration of spiral, (c) $G A$ using 25 generations, (d) $G A$ using 150 generations and (e) Division Algorithm. 


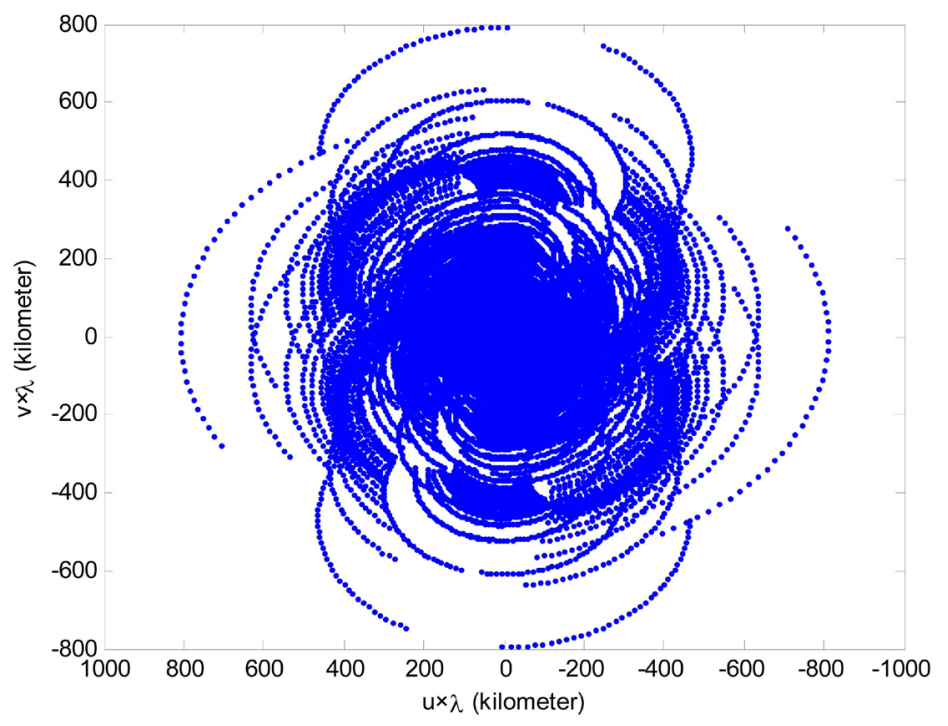

(a)

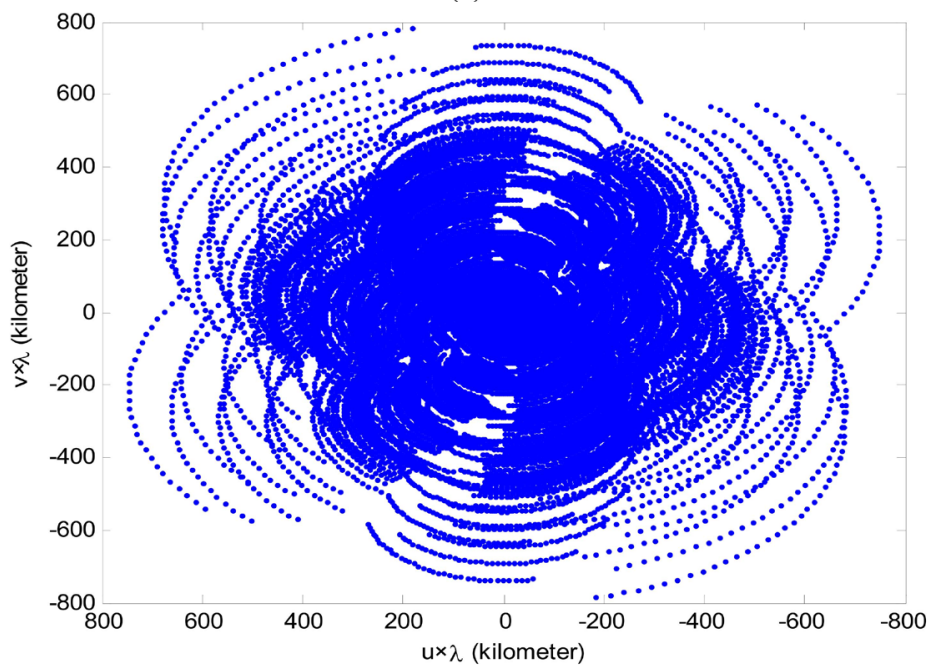

(b)

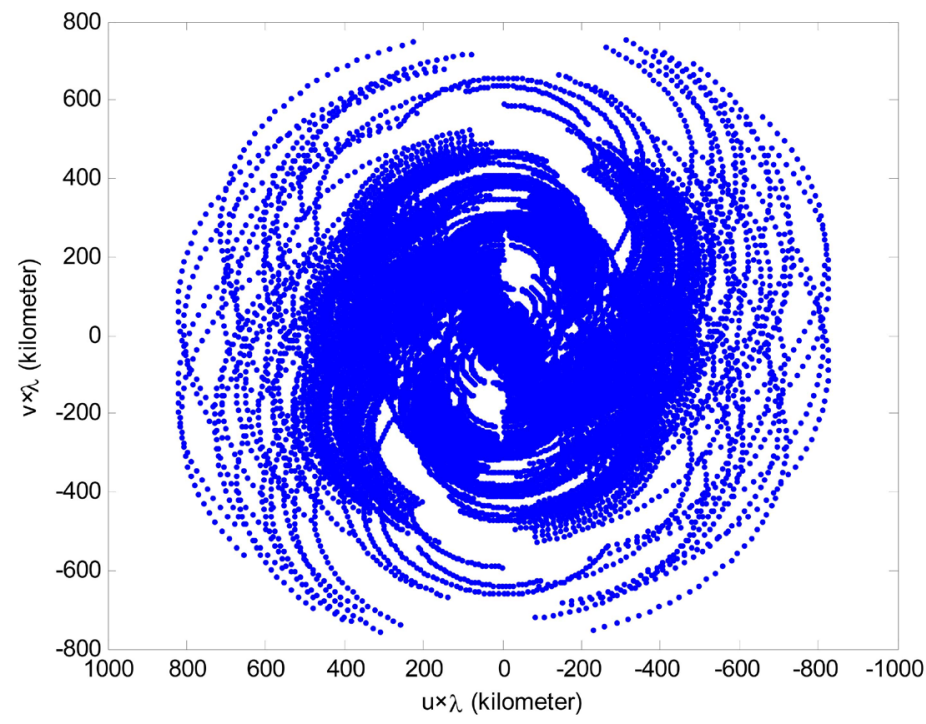

(c) 


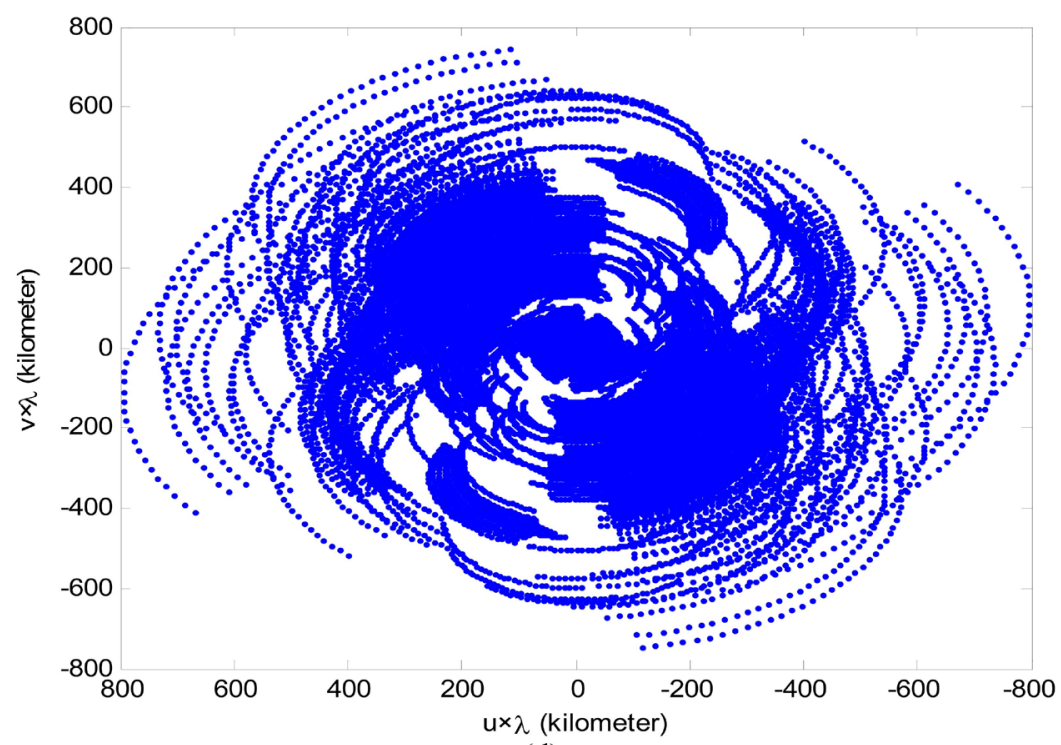

(d)

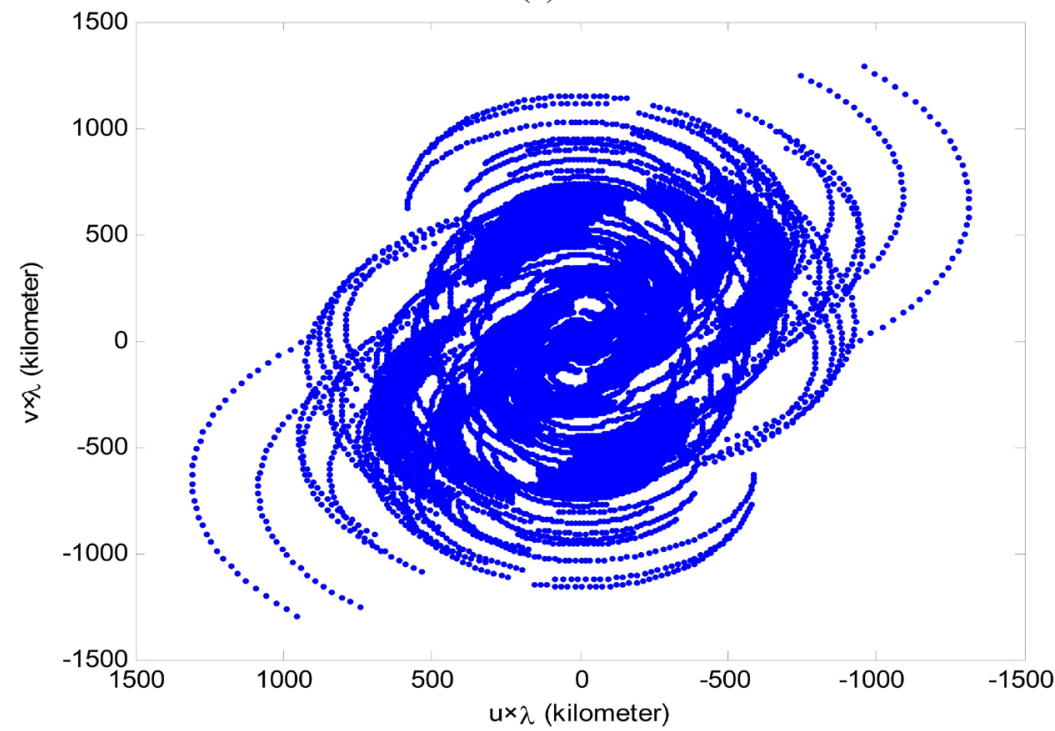

(e)

Figure 3. Spatial frequency coverage for a 6-hour tracking observation $u$ - $v$ plane coverage for configuration of (a) extended GMRT, (b) spiral, (c) twenty-five generations, (d) one hundred fifty generations using Genetic Algorithm and (e) Division Algorithm.

Table 1. Comparison of different configurations.

\begin{tabular}{ccccc}
\hline Configuration & $\begin{array}{c}\text { Overlapped } \\
\text { samples\% } \\
\text { (Snapshot) }\end{array}$ & $\begin{array}{c}\text { Overlapped } \\
\text { samples\% } \\
\text { (Hour tracking) }\end{array}$ & $\begin{array}{c}\text { Unsampled cells\% } \\
\text { (Snapshot) }\end{array}$ & $\begin{array}{c}\text { Unsampled cells\% } \\
\text { (Hour tracking) }\end{array}$ \\
\hline Extended GMRT & 89.19 & 88.89 & 78.67 & 75.19 \\
Spiral & 86.77 & 85.51 & 72.56 & 67.59 \\
25th generation & 85.91 & 86.58 & 76.34 & 70.74 \\
150th generation1 & 85.05 & 84.76 & 74.56 & 66.65 \\
DA array & 79.45 & 83.87 & 72.00 & 63.76 \\
\hline
\end{tabular}

Notes: 6-hour is used in hour tracking synthesize. 
Table 2. Comparison of GMRT's SLL and different configurations' SLL.

\begin{tabular}{cccc}
\hline Configuration & $\begin{array}{c}\text { First } S L L(\mathrm{~dB}) \\
\text { (Hour tracking) }\end{array}$ & $\begin{array}{c}\text { mean } S L L(\mathrm{~dB}) \\
\text { (Hour tracking) }\end{array}$ & $\begin{array}{c}\text { Peak } S L L(\mathrm{~dB}) \\
\text { (Hour tracking) }\end{array}$ \\
\hline Extended GMRT & -11.18 & -11.97 & -10.59 \\
Spiral & -11.25 & -12.46 & -10.86 \\
25th generation & -11.88 & -13.85 & -12.55 \\
150th generation1 & -12.04 & -13.88 & -13.75 \\
$D A$ array & -19.30 & -16.86 & -14.80 \\
\hline
\end{tabular}

Notes: 6-hour is used in hour tracking synthesize.

Table 1 shows the number of overlapped samples using snapshot as well as for a 6-hour tracking observation. This parameter is valued at $89.19 \%$ using the snapshot and $88.89 \%$ for a 6 -hour tracking observation. The unsampled cells ratio achieved with this configuration (expanded GMRT) are 78.67\% using the snapshot observation and $75.19 \%$ for a 6-hour tracking observation.

As shown in Figure 2(a), for the $u-v$ plane coverage using the snapshot observation, the linear ridges are not smooth, and this configuration provides a poor sensitivity and consequently a poor signal to noise ratio [1]. Therefore, the linear ridges need to be smoothened. In order to perform this requirement, the arms are curved in the next arrangement as is shown in Figure 2(b).

Arms in the spiral configuration cover the angular position of 60 to 96,180 to 216 and 300 to 336 degrees. The angular positions of five added antennas in each arm are 62 to 70,182 to 190 and 302 to 310 degrees. The overlapped sample ratio is equal to $86.77 \%$ for the spiral using the snapshot observation. Similarly, it is equal to $85.51 \%$ for a 6 -hour tracking observation. The unsampled cells ratio from the $u-v$ coverage indicate the percentage of the cells without any sample. This ratio is equal to $72.56 \%$ using the snapshot (see Figure 2(b)) and $67.59 \%$ for a 6-hour tracking observation (see Figure $3(\mathrm{~b})$ and Table 1). This means the spiral configuration is able to sample the Fourier space of the image better than the extended Y-shape. In comparison to the extended GMRT, the lower ratios of overlapped samples and unsampled cells resulting from the spiral suggest that this configuration provides more information about the source due to a greater number of samples in the $u-v$ plane. Therefore, the configuration of the spiral provides a better $u-v$ plane coverage in both types of observations in comparison to the extended GMRTs as shown in Figure 2(b) and Figure 3(b).

In the next step, the $G A$ is applied to this extended interferometric array, to investigate the effect of the algorithm.

From the results achieved using the previous configurations (see Figure 1(a) and Figure 1(b)), the data in the spatial frequency domain needs to be spread out to get less overlapping. Therefore, the $G A$ is applied to work on sampled data using the snapshot to provide the desired resolution.

For the $G A$, the optimum ratio values of mutation and crossover (25\% mutation ratio and $25 \%$ crossover ratio) are used in the proposed algorithm. The 
number of antennas (chromosomes) is set to 45 and for each the diameter is equal to $45 \mathrm{~m}$ to assess the efficiency of the algorithm. To distribute the sampled data in $u-v$ plane coverage more smoothly, the algorithm works on 150 generations to optimize the image resolution.

The position of the antennas (chromosomes) in the array for the $25^{\text {th }}$, and the $150^{\text {th }}$ generations using the $G A$ are shown in Figure $1(\mathrm{c})$ and Figure $1(\mathrm{~d})$, respectively. The snapshot in the $u-V$ plane coverage for the $25^{\text {th }}$, and the $150^{\text {th }}$ generations that are shown in Figure 2(c) and Figure 2(d). These illustrate how the sampled data distribution are from the results using the extended GMRT. As can be observed qualitatively, the data in the $150^{\text {th }}$ generation improves the distribution of the data somewhat more evenly. The calculated ratios shown in Table 1 summarize the ability of the $G A$ (when using snapshot) in distributing the samples and obtaining more samples rather than the extended $G M R T$.

The calculated ratios in Table 1 show that the $G A$ can sample data with less overlapped data using the snapshot observation. Specifically, for the $150^{\text {th }}$ generation the ratio is equal to $(85.05 \%)$, in comparison to $89.19 \%$ for the extended GMRT. This indicates that as the algorithm works with more generations, it distributes sampled data in the $u-v$ plane coverage more efficiently than extended $Y$-shaped.

The calculated ratio of overlapped samples for a 6-hour tracking is shown in Table 1 . From the results, this value is improved from $88.89 \%$ to $86.58 \%$, and then to $84.76 \%$ using the extended $G M R T$, the $25^{\text {th }}$, and the $150^{\text {th }}$ generation configurations, respectively. It means the algorithm improves the ratio of overlapped samples since it works with a greater number of generations.

When the number of generations goes up, the number of unsampled cells is lower; specifically, this percentage is $78.67 \%$ using the extended GMRT configuration and becomes $76.34 \%$, and $74.56 \%$ values using the $25^{\text {th }}$, and $150^{\text {th }}$ generations using the snapshot, respectively. This ratio is equal to $75.19 \%$ using the extended GMRT observation and is equal to $70.74 \%$ and $66.65 \%$ values using the $25^{\text {th }}$, and the $150^{\text {th }}$ generations for 6-hour tracking observations, respectively.

In the last step, the $D A$ is applied to this extended interferometric array, to investigate the effect of the algorithm. The algorithm provides an optimum solution for both the snapshot and for the hour-tracking observations with minimum ratios of overlapping.

The position of the antennas, $u-V$ coverages at snapshot and 6-hour tracking observations in the array using $D A$ are shown in Figure 1(e), Figure 2(e), and Figure 3(e), respectively. The calculated parameters shown in Table 1 express the ability of the $D A$ in distributing the samples and obtaining more samples rather than the extended GMRT's at the snapshot.

The results calculated in Table 1 show that the $D A$ can achieve the sampled data with less overlapped data at snapshot observation among all discussed configurations in this study (79.45\%). The calculated overlapped samples ratio for a 6 -hour tracking is shown in Table 1. From the results, this value is equal to $83.87 \%$. It means the algorithm improves the overlapped number of samples 
more efficiently than $G A$. When the number of generations goes up, the unsampled cells get reduced; specifically, this percentage is $72 \%$ at snapshot and $63.76 \%$ for a 6 -hour tracking observation.

The calculated first $S L L$, mean of the first three $S L L$ (mean $S L L$ ), and the worst $S L L$ defined as the peak $S L L$ are shown in Table 2. The calculated $S L L$ s show that the spiral geometry has lower side lobes $(S L L=-11.25 \mathrm{~dB}$ and mean $S L L=$ $-12.46 \mathrm{~dB}$, and peak $S L L=-10.86 \mathrm{~dB})$ than the extended $G M R T(S L L=-11.18$ $\mathrm{dB}$ and mean $S L L=-11.97 \mathrm{~dB}$, and peak $S L L=-10.59 \mathrm{~dB})$ and the linear ridges using the snapshot is also smoother than for the extended GMRT (see Figure 2(b)). As such, the spiral configuration smoothens the linear ridges.

The first and the mean values of the first three $S L L$ s of $25^{\text {th }}$ and $150^{\text {th }}$ generation using $G A$ are also calculated Table 2 . The first $S L L$ is $-11.18 \mathrm{~dB}$ in extended GMRT and $-11.88 \mathrm{~dB}$, and $-12.04 \mathrm{~dB}$ for the $25^{\text {th }}$, and the $150^{\text {th }}$ generation configurations, respectively. The calculated mean value of the first $S L L$ shown in Table 2 depicts that the configurations have the mean $S L L$ of $-13.85 \mathrm{~dB}$ and $-13.88 \mathrm{~dB}$ at the $25^{\text {th }}$, and the $150^{\text {th }}$ generation, respectively (this ratio is valued at $-11.97 \mathrm{~dB}$ using the extended GMRT). The algorithm is also able to decrease the level of the peak $S L L$ as the number of generations goes up (this ratio is $-12.55 \mathrm{~dB}$ and $-13.75 \mathrm{~dB}$ using $25^{\text {th }}$ and $150^{\text {th }}$ generation configurations, respectively). The calculated first $S L L$, mean $S L L$, and peak $S L L$ values of $D A$ configuration are $-19.3 \mathrm{~dB},-16.86 \mathrm{~dB}$, and $-14.8 \mathrm{~dB}$, respectively. These values show the better efficiency of the $D A$ in comparison to the $G A$.

The evolution of the average fitness in each generation for the $u-v$ plane coverage using the snapshot observation and $S L L$ reduction are shown in Figure 4. As shown in Figure 4(a), the optimum solution occurred at the $47^{\text {th }}$ generation for the first 50 generations, and the optimum value in the range of $82^{\text {th }}$ to $150^{\text {th }}$

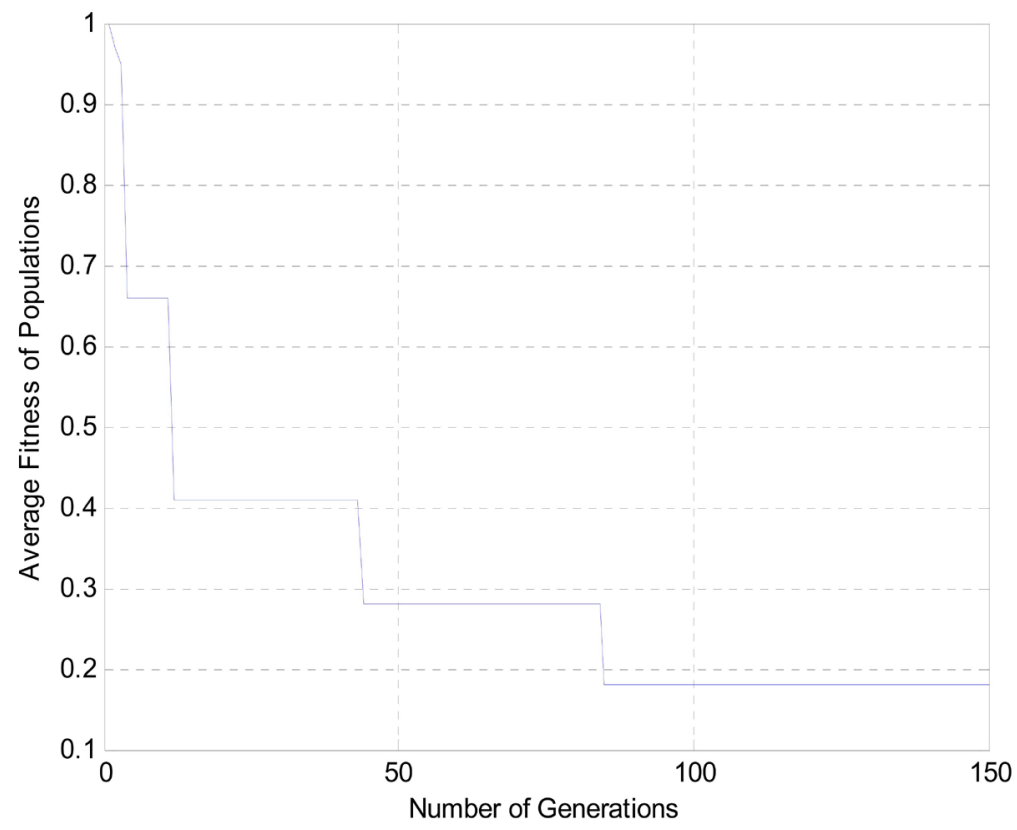

(a) 


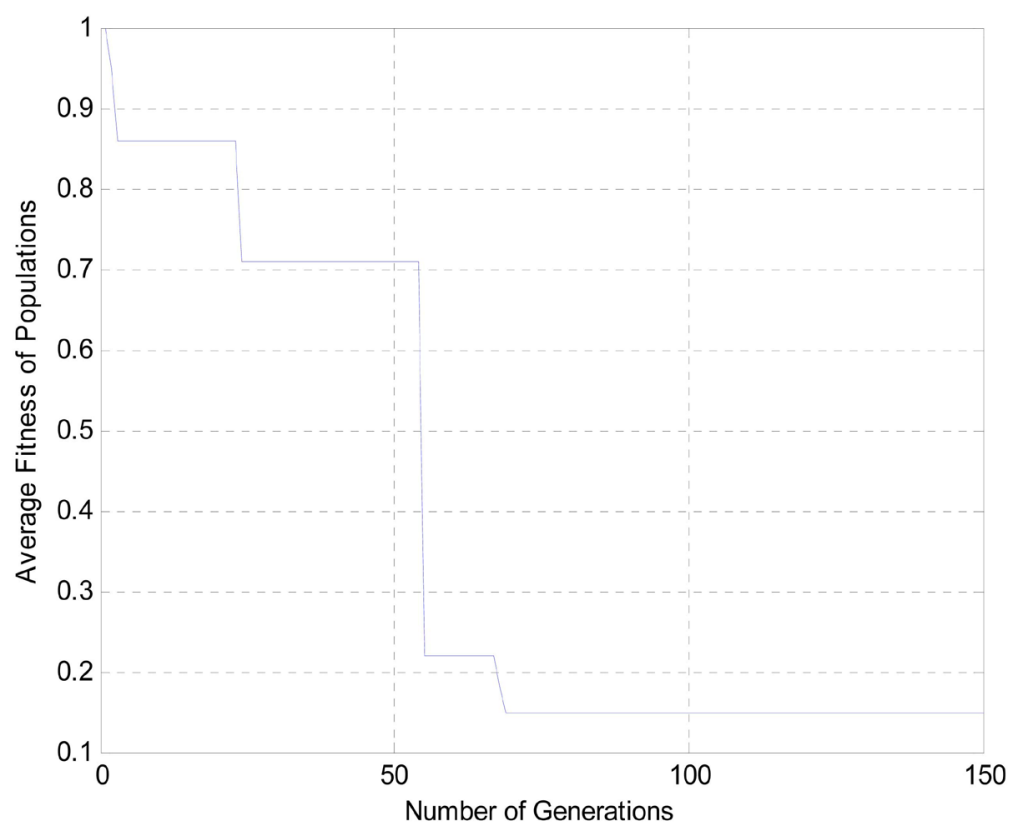

(b)

Figure 4. The evolution of average fitness in each generation in (a) the spatial frequency domain and (b) the l-m domain.

generation remains constant. The fitness value for the first 50 generations in Figure 4 (b) obtains the optimum solution at $23^{\text {rd }}$ generation. Since the algorithm seeks the solutions randomly, it provides different solutions each time. The aim of this study is to demonstrate optimum solutions for an extended interferometric array. It is not written for any specific constraints for astronomy applications; however, it can work with different constraints.

From the results obtained from the different configurations in this study, a spiral shape suggests good results in the angular domain as well as in the $u-V$ domain and the $G A$ and $D A$ provide improved $S L L$ s, and $u-v$ plane coverages.

\subsection{Antenna Array in Malaysia}

The simulated source declination is 45 degrees. The duration for the fully synthesized observation is a 6-hour tracking with 10 minutes time interval between each two samples.

As a first step, the $G A$ is applied to an interferometric array, to investigate the effect of the algorithm.

The algorithm provides an optimum solution for both the snapshot and the hour-tracking observations with minimum values of overlapping (this happens due to the grounding of the plane as defined in (2) and (3)).

The optimum ratio values of mutation and crossover (25\% mutation ratio and $25 \%$ crossover ratio) are used in the algorithm. The number of antennas (chromosomes) is fixed to 10 . To distribute the sampled data in $u-v$ plane coverage more smoothly, the $u-v$ plane is gridded with the dimension of $D_{u} \times D_{v}$ as defined in (3) and the algorithm works on 150 generations to optimize the resolu- 
tion of the image.

The positions of the antennas (chromosomes) in the array of the $25^{\text {th }}$ and $150^{\text {th }}$ generations using the $G A$ are shown in Figure 5(a) and Figure 5(b), respectively. Using the snapshot, the $u$ - $v$ plane coverages for the $25^{\text {th }}$, and $150^{\text {th }}$ generations in Figure 6(a) and Figure 6(b) and 6-hour tracking in Figure 7(a) and Figure 7 (b) show how the sampled data is distributed using $25^{\text {th }}$ generation gradually to $150^{\text {th }}$ generation.

The calculated parameters shown in Table 3 express the ability of the $G A$ to distribute the samples and obtaining more samples as the number of generations goes up at the snapshot. The calculated results summarized in Table 3 show that the $G A$ achieves the sampled data with less overlapped data at snapshot observation from the $25^{\text {th }}$ generation $(12 \%)$ to the $150^{\text {th }}$ generation $(2 \%)$. This indicates

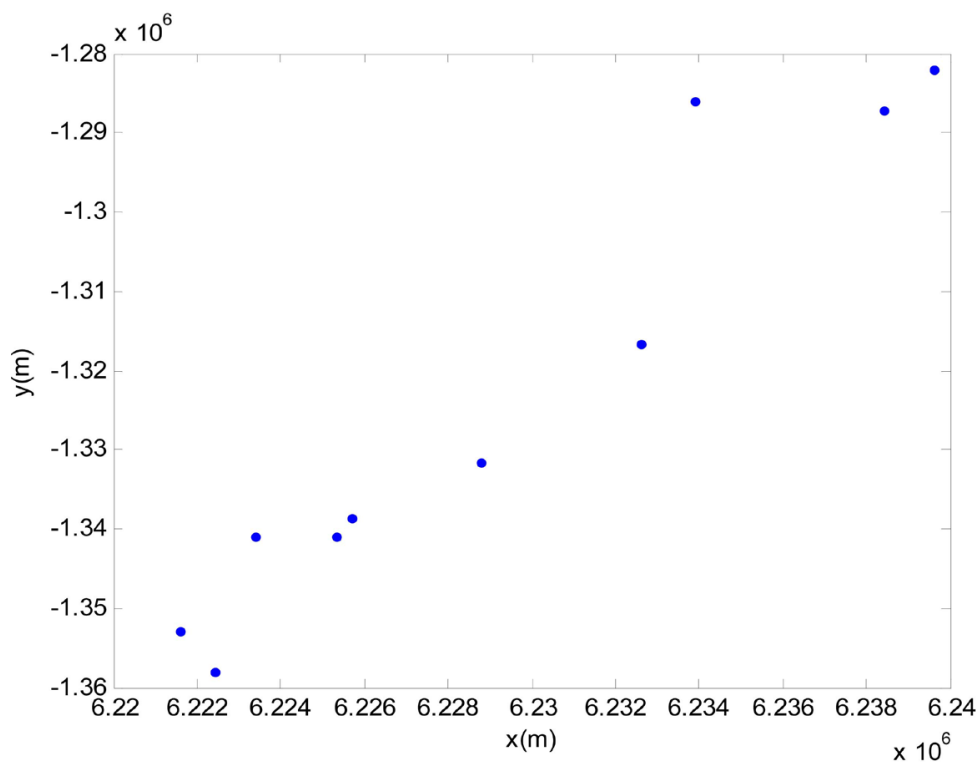

(a)

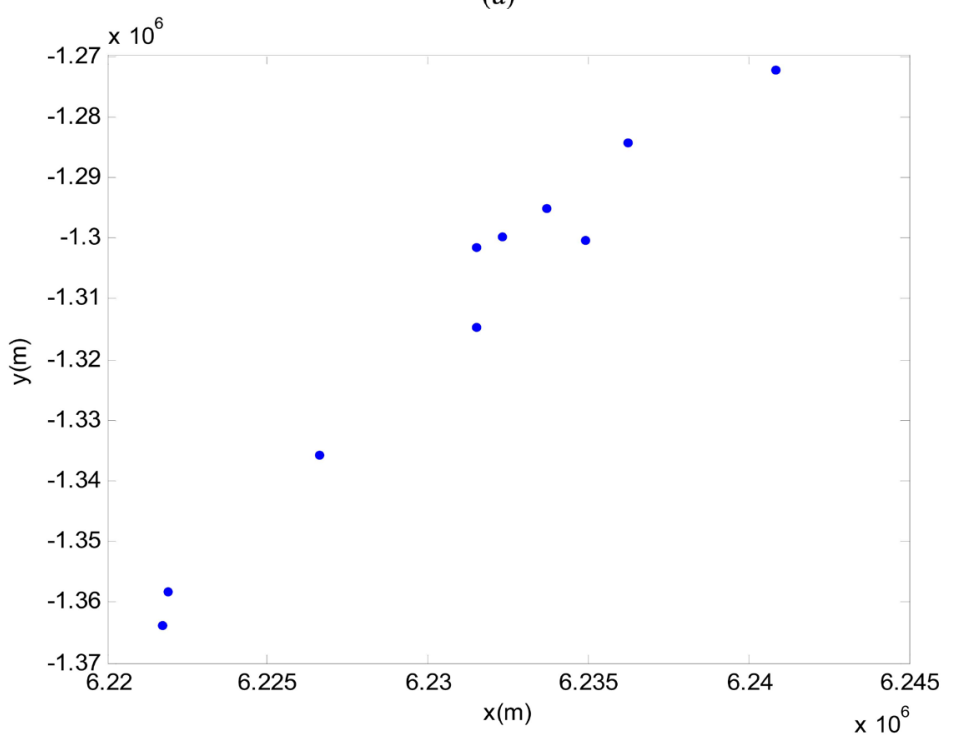

(b) 


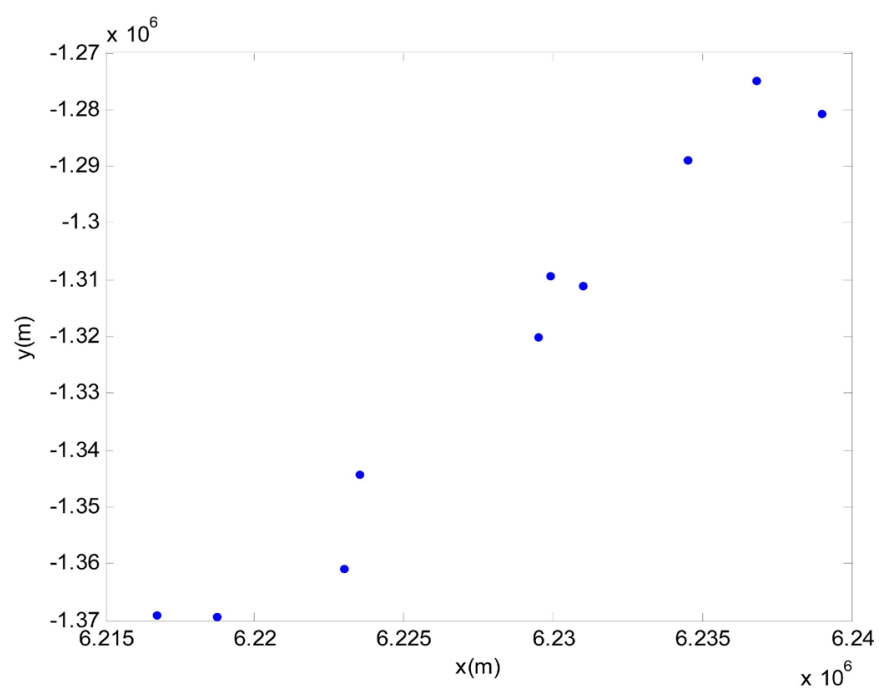

(c)

Figure 5. Configuration for (a) twenty-five generations, (b) one hundred fifty generations using Genetic Algorithm and (c) Division Algorithm.
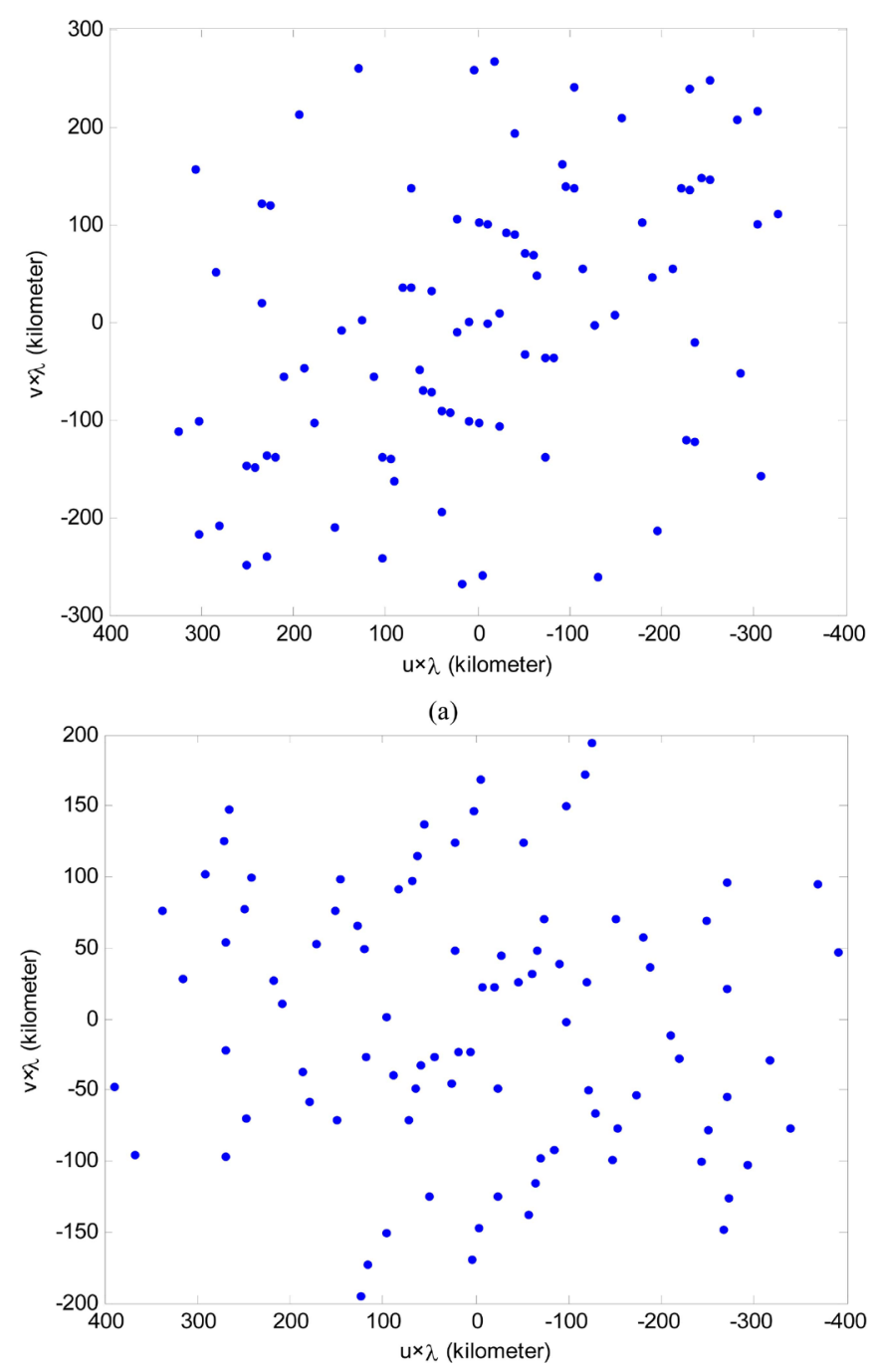

(b) 


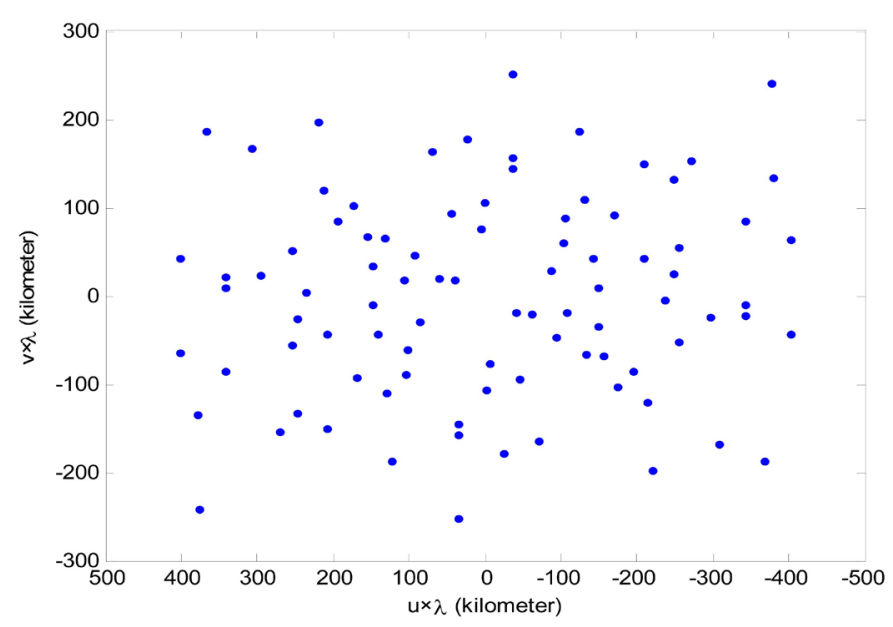

(c)

Figure 6. Snapshot $u-v$ plane coverage for (a) twenty-five generations, (b) one hundred fifty generations using Genetic Algorithm and (c) Division Algorithm.

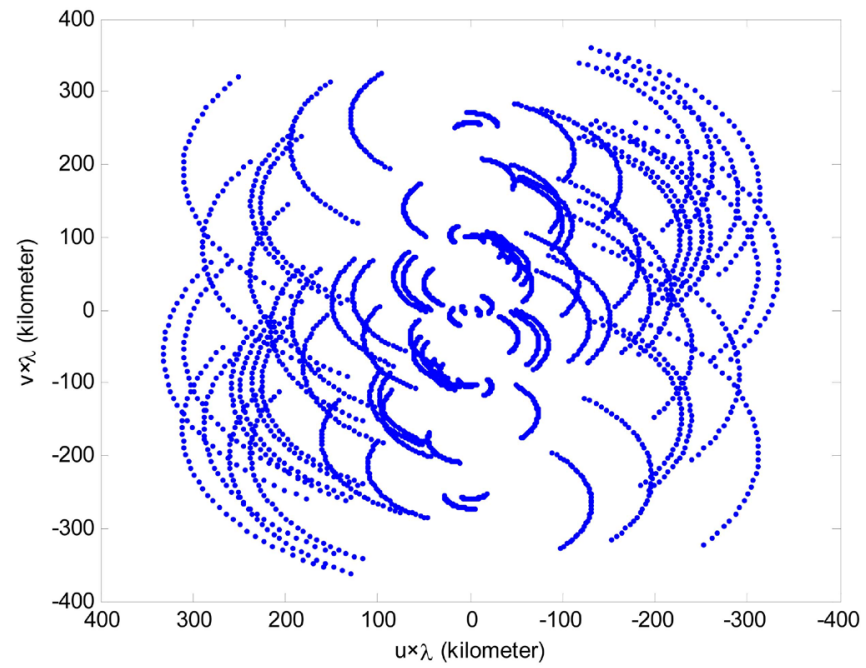

(a)

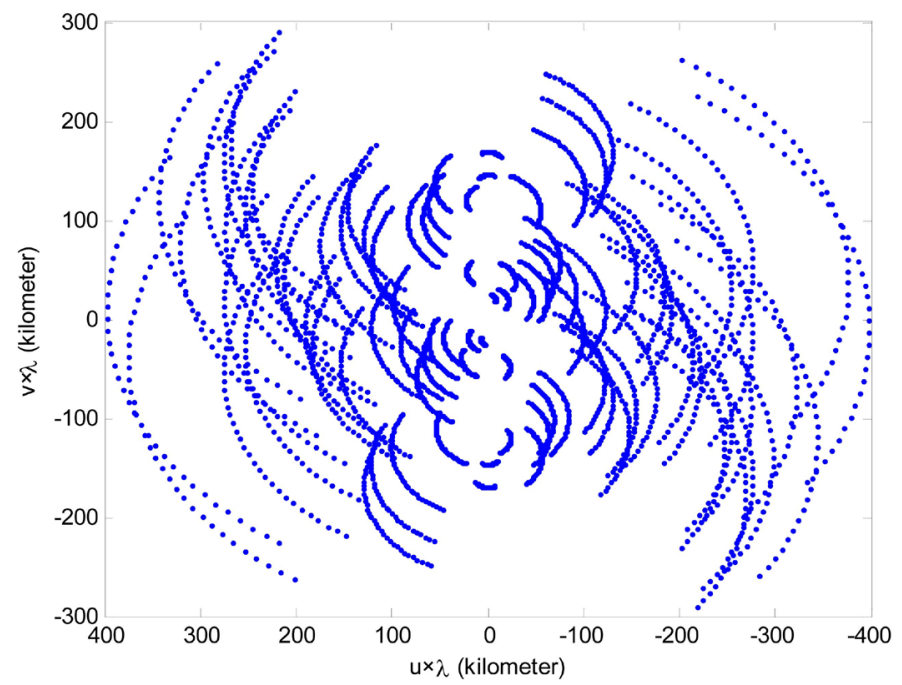

(b) 


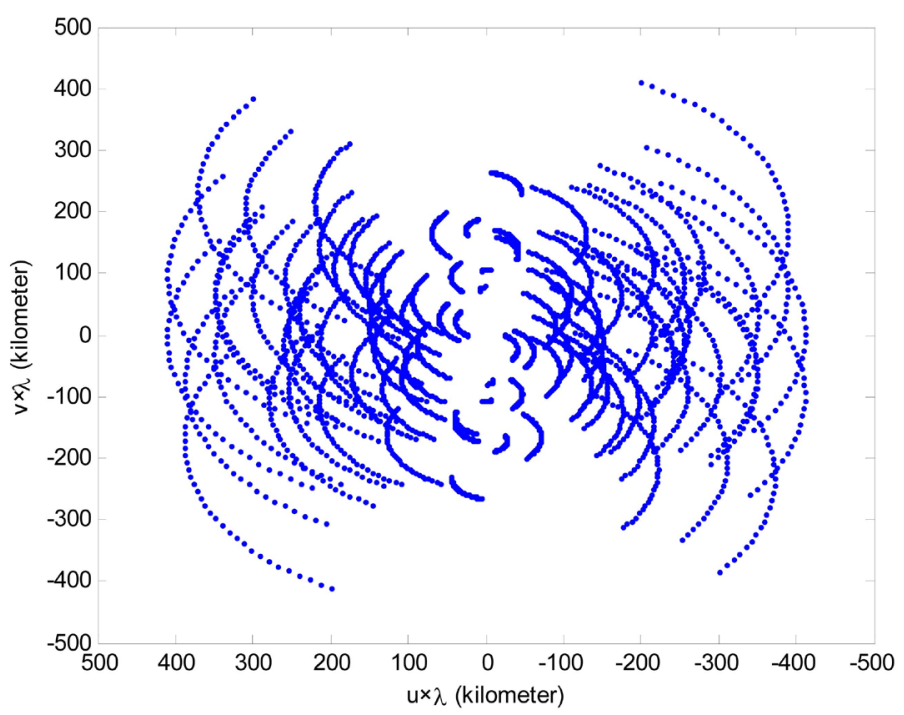

(c)

Figure 7. Spatial frequency coverage for a 6-hour tracking observation $u$ - $v$ plane coverage for (a) twenty five generations, (b) one hundred fifty generations using Genetic Algorithm and (c) Division Algorithm.

Table 3. Comparing of different parameters of $D A$ to the $G A$.

\begin{tabular}{ccccc}
\hline Configuration & $\begin{array}{c}\text { Overlapped } \\
\text { samples\% } \\
\text { (Snapshot) }\end{array}$ & $\begin{array}{c}\text { Overlapped } \\
\text { samples\% } \\
\text { (Hour tracking) }\end{array}$ & $\begin{array}{c}\text { Unsampled cells\% } \\
\text { (Snapshot) }\end{array}$ & $\begin{array}{c}\text { Unsampled cells\% } \\
\text { (Hour tracking) }\end{array}$ \\
\hline 25th generation & 13.34 & 25.23 & 94.89 & 93.23 \\
150th generation1 & 2.23 & 24.45 & 94.00 & 92.49 \\
$D A$ array & 0.00 & 22.28 & 91.56 & 92.16 \\
\hline
\end{tabular}

Notes: 6-hour is used in hour tracking synthesize.

that as the algorithm works with more generations, it can distribute sampled data in the $u$-v plane coverage more efficiently.

The calculated overlapped samples ratio for a 6-hour tracking is shown in Table 3. From the results, this value is varied from $13.34 \%$ to $2.23 \%$ using the $25^{\text {th }}$ and the $150^{\text {th }}$ generation configurations, respectively. It means the algorithm improves the overlapped samples as it works with more numbers of generations.

Since the number of generations goes up, the number of unsampled cells are decreased; specifically, the ratio is $94.89 \%$ using the $25^{\text {th }}$ generation observation and becomes $94.00 \%$ using the $150^{\text {th }}$ generations at the snapshot. This ratio is equal to $93.23 \%$ using the $25^{\text {th }}$ generation observation and becomes $92.49 \%$ using the $150^{\text {th }}$ generation for a 6 -hour tracking observation.

Finally, the $D A$ is applied to the extended interferometric array, to investigate the effect of the algorithm. The algorithm provides an optimum solution for both the snapshot and the hour-tracking observations with minimum values of overlapping (this happens due to the gridding of the plane as defined in (2) and (3)).

The position of the antennas, the $u-V$ coverage for the snapshot observation and for a 6-hour tracking observation in the array using the $D A$ are shown in 
Figure 6(c), Figure 7(c), and Figure 8(c), respectively. The calculated ratios shown in Table 3 express the ability of the $D A$ in distributing the samples and obtaining more samples rather than the $G A$ for the snapshot.

The calculated results that are summarized in Table 3 show that the $D A$ can achieve the sampled data with the same efficiency as the $G A$ for the snapshot observation. The calculated overlapped samples ratio for a 6-hour tracking is shown in Table 3. From the results, this ratio is valued at $22.28 \%$. It means the algorithm improves the overlapped samples more efficiently than $G A$ for a 6-hour tracking observation.

Since the number of generations goes up, the number of unsampled cells is reduced; specifically, this percentage is $91.56 \%$ at snapshot and $92.16 \%$ for a 6-hour tracking observation, which show the same efficiency of the $G A$.

The calculated first $S L L$, mean values of the first three $S L L$ s (mean $S L L$ ), and the worst $S L L$ defined as the peak $S L L$ are shown in Table 4 . The values of the

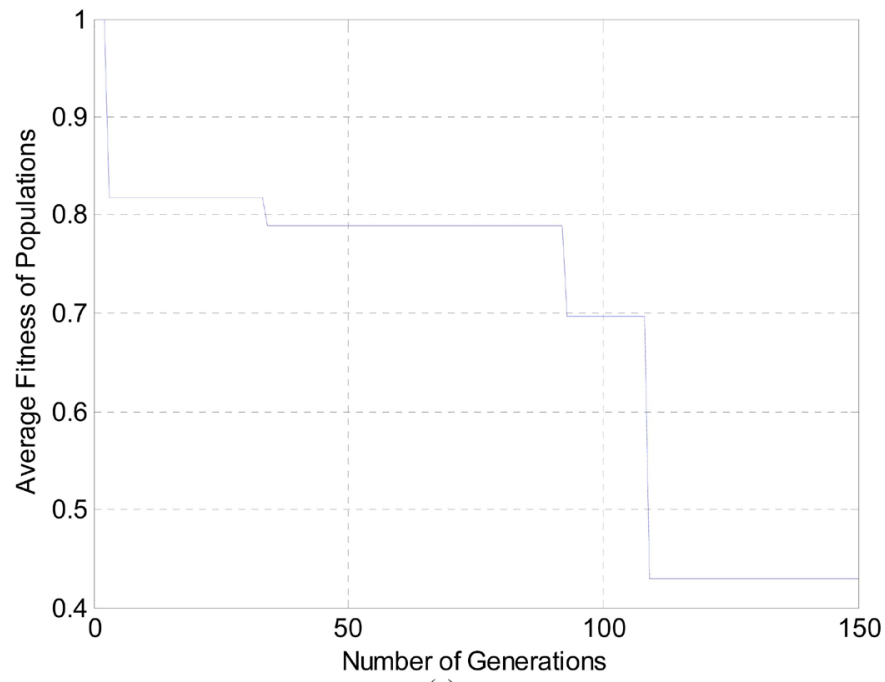

(a)

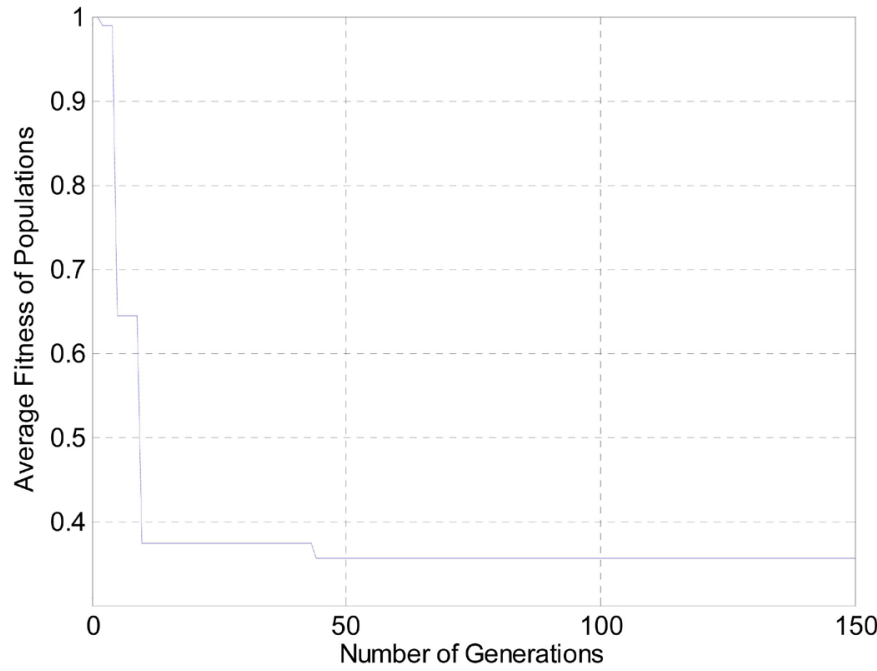

(b)

Figure 8. The evolution of average fitness in each generation in (a) the spatial frequency domain and (b) the l-m domain. 
Table 4. Comparison of different configurations.

\begin{tabular}{cccc}
\hline Configuration & $\begin{array}{c}\text { First } S L L(\mathrm{~dB}) \\
\text { (Hour tracking) }\end{array}$ & $\begin{array}{c}\text { mean } S L L(\mathrm{~dB}) \\
\text { (Hour tracking) }\end{array}$ & $\begin{array}{c}\text { Peak } S L L(\mathrm{~dB}) \\
\text { (Hour tracking) }\end{array}$ \\
\hline 25th generation & -6.99 & -6.75 & -3.03 \\
150th generation1 & -5.23 & -8.45 & -5.20 \\
$D A$ array & -10.00 & -9.86 & -5.88 \\
\hline
\end{tabular}

Notes: 6-hour is used in hour tracking synthesize.

first $S L L$, mean $S L L$, and peak $S L L$ are $-6.99 \mathrm{~dB},-6.75 \mathrm{~dB}$, and -3.03 using the $25^{\text {th }}$ generation, $-5.23 \mathrm{~dB},-8.45 \mathrm{~dB}$, and $-5.2 \mathrm{~dB}$ using the $150^{\text {th }}$ generation, and $-10 \mathrm{~dB},-9.86 \mathrm{~dB}$, and $-5.88 \mathrm{~dB}$ for the $D A$ array, respectively. It shows that even though the $G A$ decreases the $S L L$, the $D A$ gains reasonable $S L L$ and optimum ratios in the spatial frequency domain, with the same population.

The evolution of the average fitness in each generation for the $u$ - $v$ plane coverage at the snapshot and $S L L$ reduction is shown in Figure 8. As shown in Figure 8 (a), for the first 50 generations, the optimum solution is reached at the $38^{\text {th }}$ generation. In contrast, for a total of 150 generations, a better solution is obtained at the $112^{\text {th }}$ generation. Similarly, the fitness value for the first 50 generations in Figure 8 (b) obtains the optimum solution at $43^{\text {th }}$ generation. Since, the algorithm seeks for the solutions randomly; it provides different solutions for each generation.

\section{Discussion}

Astronomical observations benefit from arrays that can achieve high resolution with low $S L L s$, smooth linear ridges, and good $u-v$ plane coverage. The aim of the study was to extend such an array to optimize the configuration. Therefore, the principal aim of the present simulation was to compare different extended configurations. Based on the results shown in Table 1, the spiral configuration or curved arm achieves good results in 1-m domain and $u-v$ domain. Also, it was found that the curvature smoothens the linear ridges for a low duration observation. Finally, the $G A$ was able to provide good results for all the desired requirements.

Malaysia has started to get involved in astronomy project recently with two telescopes. As such, the second part of this study has investigated an optimum solution for the future correlator array antennas in Malaysia. Based on the results, it has been shown that the $D A$ is able to obtain a configuration that provides almost all desired requirements in both spatial frequency domain and angular domain.

\section{Conclusions}

In conclusion, the aim of this study was to investigate different solutions to extend an interferometric array and the future array in Malaysia. For expanding the existing array, initially, the expansion along the three arms configuration was 
studied and then the effect of expanding it to a spiral shape was evaluated. The results have shown that the spiral could provide better $u$ - $v$ plane coverage in a 6-hour tracking synthesized observations (in an aperture synthesis observation of six hours duration) with the lowest levels of the $S L L$. Then, the $G A$ was applied to the interferometric array. From the results (different results of $u$ - $v$ plane coverage) shown in this paper, the extended curved arms have better $u-v$ coverage results than the extended $Y$-shaped. It also suppressed the $S L L$ s. As such, the algorithm improves the number of overlapped samples, as the number of generations increases. Finally, the $D A$ was applied to such an array. Calculated results in Table 1 show that the $D A$ method can sample the data more efficiency for the snapshot observation compared to the other configurations discussed in this paper.

Then, the $G A$ and $D A$ were applied to 10 antennas. Calculated results show that the $D A$ can achieve the sampled data with the same efficiency as the $G A$ for the snapshot observation. The calculated overlapped samples ratios for a 6-hour tracking are discussed. It is shown that the $D A$ improves the overlapped samples ratio more efficiently than the $G A$ for a 6-hour tracking observation.

\section{Conflicts of Interest}

The authors declare no conflicts of interest regarding the publication of this paper.

\section{References}

[1] Shahideh, K., Cada, M., Chen, Z., Shahabi, A., Short, C.I., Abidin, Z.Z. and Samiramis, K. (2018) Astronomical Journal, 156, 177.

[2] Shahideh, K., Noordin, N.K., Sali, A. and Zamri, Z.A. (2014) Astronomical Journal, 147,147

[3] Shahideh, K., Valente, D., Cada, M., Noordin, N.K. and Shahabi, A. (2017) Astronomical Journal, 154, 167.

[4] Shahideh, K., Kyun, Ng.C. and Noordin, N.K. (2013) Expansion of a Y-Shaped Array Antennas for Radio Astronomy. 2013 IEEE International Conference on Space Science and Communication (IconSpace), Melaka, 1-3 July 2013, 39. 